\title{
Underground Pipelines Wireless Sensor LNA Circuit Design and Simulation Based On ADS
}

\author{
Zhengshan Xu, Changwen Liu, Tao Chen, Fan Yang and Xu Wang \\ Beijing institute of Radio Metrology and Measurements Beijing, China
}

\begin{abstract}
LNA (Low-noise amplifier) is RF front-end major device for receiving the signal, which is used to amplify the signal close to noise. Stability, gain, input and output VSWR and noise are the most important factors to be considered in the LNA actual design. In this paper, we use the ADS software for the low noise amplifier S-parameter simulation. LNA design requires a good stability in the operating frequency band i.e. the stability factor is greater than 1 , the gain is approximately $20 \mathrm{~dB}$, the input VSWR less than 1.5, the output VSWR less than 2; In the practical design of underground pipelines wireless sensor, the result of simulation is stable and reliable.
\end{abstract}

Keywords-LNA;ADS; S-parameter; VSWR ;gain; noise

\section{INTRODUCTION}

With the development of the smart pipe, the terminal equipment and sensor devices are showing explosive growth trend. Each sensor is a source of information, collected environmental data and send it in a certain frequency, for example 433MHz. Low-noise amplifier is major device for receiving the signal in RF front-end circuit, which is used to amplify the signal close to noise. Stability, gain, input and output VSWR and noise are the most important factors to be considered in the LNA actual design. Due to the presence of the amplifier input output port reflection, under certain conditions, an amplifier no longer amplify the signal and generates the oscillation, so the stability simulation test is necessary for the amplifier design. Since the role of the amplifier is to amplify the signal, the amplifier gain must meet the design requirements. Input and output VSWR is a function of the reflection coefficient, which to a certain extent reflects the situation in the port matching of amplifier design, and the quality of its properties to a certain extent will have an impact on the stability of the low noise amplifier [1]. Due to the low noise amplifier operating at low signal state, so S-parameter simulation has practical significance for the actual circuit design of the low noise amplifier. But LNA noise figure can not be S-parameter simulation, this article does not validate noise figure. Since LNA noise figure can not be verified by Sparameter simulation, this paper does not validate noise figure.

\section{LAN PARAMETERS SIMULATION}

HMC616LP3 is used in this design, which works in $175 \mathrm{MHz}-660 \mathrm{MHz}$. HMC616LP3 is the product of Hittite, which uses $3 \mathrm{~V}-5 \mathrm{~V}$ low-voltage power supply and it's $\mathrm{I}_{\mathrm{dd}}$ can be set by $R_{\text {bias }}$. The size of the $R_{\text {bias }}$ also has an impact on the stability of LNA. In order to ensure the stability of the LNA in operating frequency, the value of $\mathrm{R}_{\text {bias }}$ should not be less than
1K. Within the operating frequency, HMC616LP3 has maximum gain up to $24 \mathrm{~dB}$ and noise figure $0.5 \mathrm{~dB}$. The stability, gain, input and output VSWR and input and output impedance data of the HMC616LP3 can be get through Sparameter simulation. HMC616LP3's S-parameter files can be downloaded in Hittite official website. The low noise amplifier HMC616LP3 ADS S-parameter simulation schematic is shown in Figure I [2].

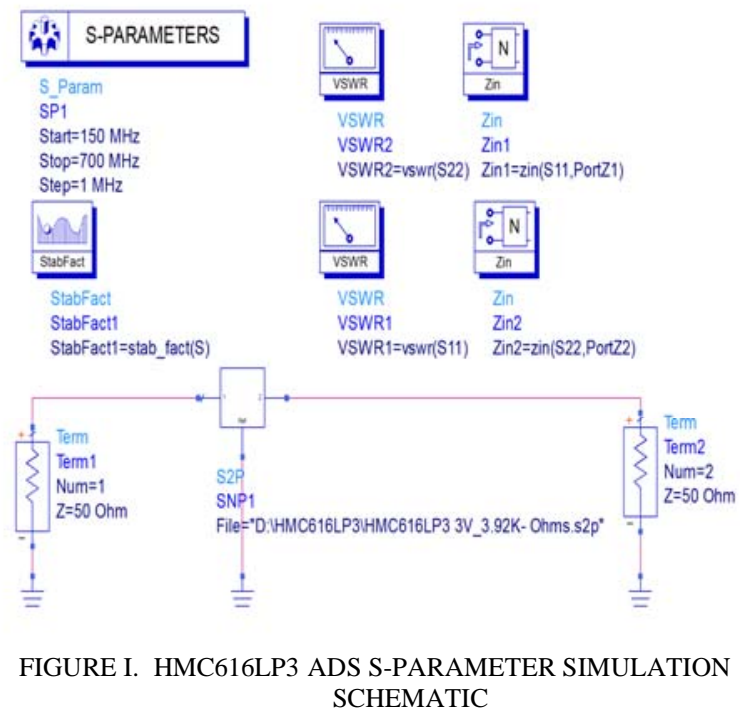

Figure I show the HMC616LP3 S-parameter simulation schematic under $3 \mathrm{~V}$ power supply, $\mathrm{R}_{\text {bias }}$ is $\mathrm{R} 3.92 \mathrm{k}$ conditions. As shown in Figure I, frequency range of the S-parameter simulation is $100 \mathrm{MHz}-1 \mathrm{GHz}$, and the simulation step is $1 \mathrm{MHz}$; The stability factor, input and output VSWR, input and output impedance parameters can be calculated by the S-parameter simulation. The low noise amplifier HMC616LP3 ADS Sparameter simulation results is shown in Figure II. 

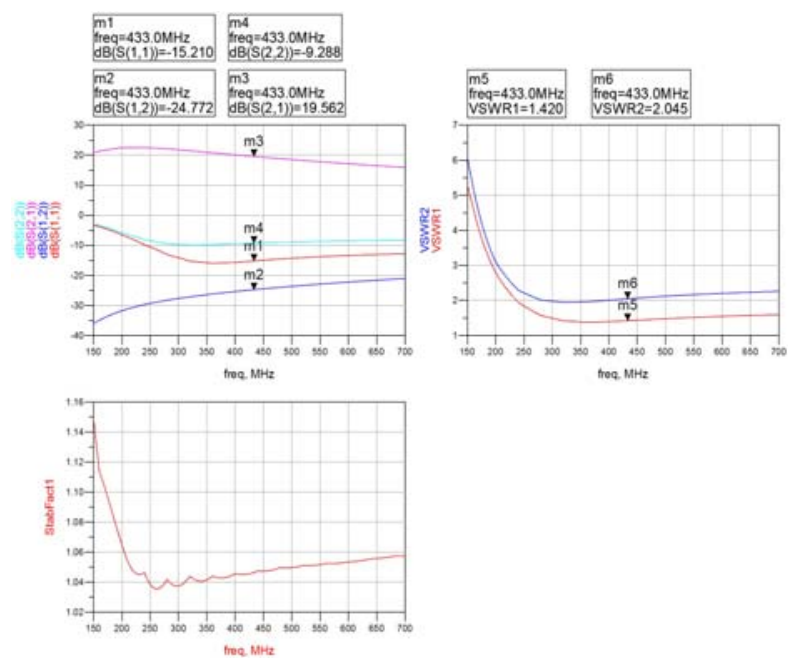

FIGURE II. THE RESULT OF HMC616LP3 S-PARAMETER SIMULATION

Shown in Figure II, the parameter S $(2,1)$ of HMC616LP3 in the $433 \mathrm{MHz}$ is $19.562 \mathrm{~dB}$, basically meet the design requirements of about 20dB. LNA input VSWR is 1.420 (less than 1.5), reached the design requirements of the input VSWR. LNA output VSWR is 2.045 (more than 2.0), did not meet the design requirements of the output VSWR. As shown in Stabilizing factor graph, in the $433 \mathrm{MHz}$ working frequency the stability factor is greater than 1, indicating that LNA is stable.

\section{OPTIMIZATION DESIGN OF LNA CICUIT}

Since the amplifier output VSWR at the operating frequency of $433 \mathrm{MHz}$ does not meet the design requirements, it is necessary to adjust the output VSWR .The output VSWR can be adjusted by adjusting the input and output impedance[3]. Figure III show the input and output impedance values of HMC616LP3 at different frequencies.

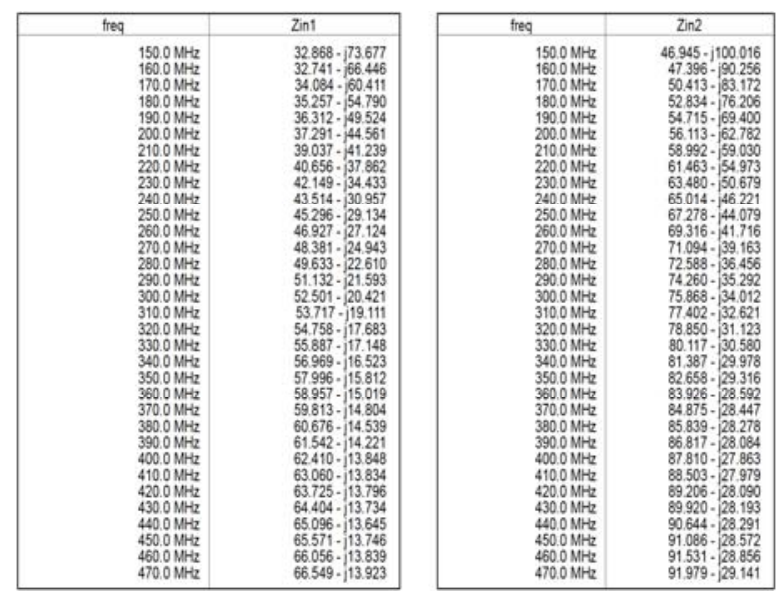

FIGURE III. INPUT AND OUTPUT IMPEDANCE VALUES OF HMC616LP3 AT DIFFERENT FREQUENCIES

As shown in Figure III, the input impedance of the LNA is 64.404-j*13.734 and the output impendence of the LNA is $89.920-\mathrm{j} * 28.193$ at the frequency of $430 \mathrm{MHz}$. Matching network can be designed by ADS Smith Chart tool as shown in FIGURE IV[4].

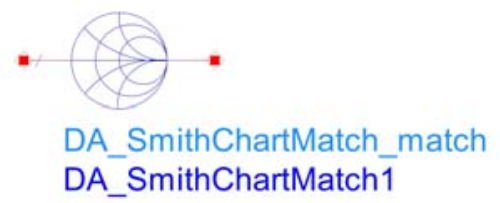

FIGURE IV. ADS SMITH CHART TOOL

ADS simulation schematic after optimization of the input impedance is shown in Figure V.

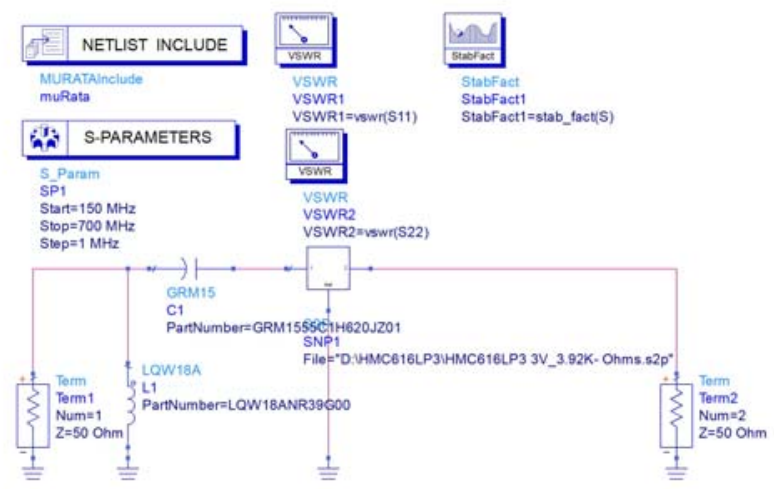

FIGURE V. OPTIMIZED ADS SIMULATION SCHEMATIC

The module of inductance and capacitance used in the simulation is provided by Murata, which has the same characteristics as the actual components.

The inductance and capacitance module used in the simulation is provided by Murata, they have the same characteristics as the actual components. So the simulation results using this model are more instructive. The simulation results after optimizing input impedance is shown in figure VI.
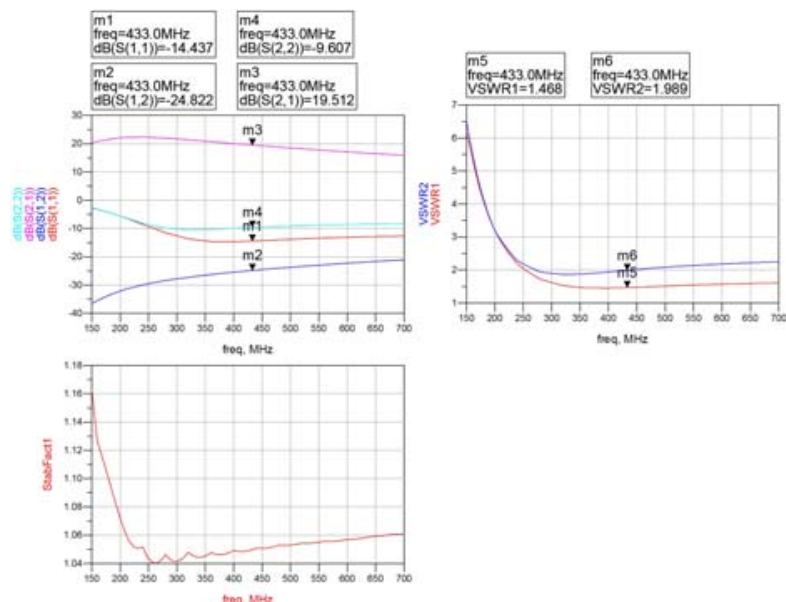

FIGURE VI. THE SIMULATION RESULTS AFTER OPTIMIZING INPUT IMPEDANCE

As shown in figure VI, at the frequency of $430 \mathrm{MHz}$ the value of output VSWR has changed into 1.898. After optimizing input impedance the output VSWR has met the 
requirement of design. At the same time, the stability factor has been improved. If the input impedance is adjusted and then yet not meet the design requirements, then the adjustment of output impedance is needed[5]. It can be optimized by ADS using 'Optim' control tool and the 'Goal' target control tool. Then it is just need to replace the simulating value with the value closest to the actual component to optimize Components, and confirm the final simulation results.

\section{CIRCUIT DESIGN OF LNA}

As shown above, after series of simulation and optimization, using the Murata GRM series 52pF capacitor and LWQ series $390 \mathrm{nH}$ inductance the actual impedance matching circuit is shown in Figure VII.

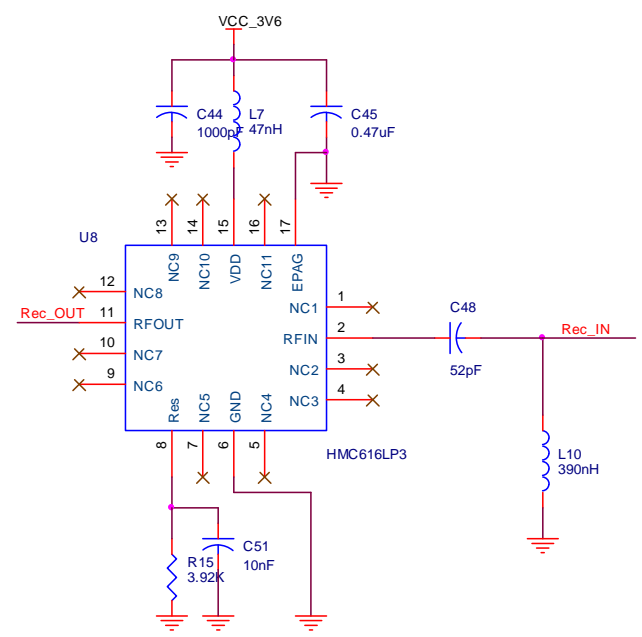

FIGURE VII. ACTUAL IMPEDANCE MATCHING CIRCUIT

\section{CONCLUSION}

This article describes the $\mathrm{S}$ parameter simulation in $\mathrm{RF}$ circuit design and the performance of circuit designed by this method is stable and reliable in the practical application of wireless sensors in underground pipelines, the actual results met the design requirements.

\section{REFERENCES}

[1] Kan Neng HU,Xi You Bao,Wang Zhong Hang. LNA design using AT41511 based on ADS[J]. ELECTRONIC MEASUREMENT TECHNOLOGY,2008,31(8):24-27.

[2] He Suqin,Bai Tiansh. Simulation and Analysis of RF Circuit Design Using ADS[J]. Microelectronics,2011, 41(4):479-483.

[3] Wu Guozeng, Yang Ying. Research of Low-noise Amplifier Matching Network design Method[J].Electronics APPlications,2007,9(1):64-66.

[4] Xu Xingfu. ADS2008 RF Circuit Design And Simulation Examples [M] Beijing: Publishing House of Electronics Industry.2009:120-125.

[5] Mou Shouxi, Ma Jianguo, Yeo Kiat Seng, etal. A modified architecture used for input matching in CMOS low-noise amplifiers[J]. IEEE Transactions on Circuits and Systems II :Express Briefs, 2005, 52(11):784-788. 